\title{
Simulation analysis of flow field in the micro cavity of sapphire based on Fluent
}

\author{
Cui Wang \\ Department of Engineering Machinery \\ Jilin Communication Polytechnic \\ Changchun, China
}

\author{
Qiming Qian, Qiang Liu, Yu Sun \\ School of mechanical science and engineering \\ Jilin University, Changchun, China \\ Corresponding author: Qiang Liu \\ Email: liuqiang2012@jlu.edu.cn
}

\begin{abstract}
When polishing the micro cavity of sapphire with abrasive flow, it is necessary to analyze the state of flow field of polishing liquid in the micro cavity to analyze the effect law of polishing abrasive on polishing. It will also influence fluid parameters and the motion trajectory of abrasive in the micro cavity when the flow velocity gets into the micro cavity. It will makes fluid parameters and the motion trajectory to be more uniform when imposing ultrasonic vibration on polished workpiece, which makes the polishing effect of micro cavity better.
\end{abstract}

\section{Keywords-Fluent, micro cavity, simulation of flow field}

\section{INTRODUCTION}

Because of the tiny structure of the micro cavity of sapphire, it cannot work to polish the inner surface of the micro cavity by traditional contact polishing. It mainly polish the micro cavity with the help of polishing liquid contain nanometer abrasive. The polishing process is mainly the impact of abrasive and the surface of materials, at the same time, it will be better for polishing to impose ultrasonic vibration on the workpiece to improve the chance of impact of abrasive and machined surface. The motion state of abrasive decides the surface roughness and polishing efficiency. However, the movement speed and the motion trajectory of abrasive depend on test parameters of polishing liquid. So it is necessary to use Fluent to analyze the effect law of polishing effect, fluid parameters and the motion trajectory of abrasive in the micro cavity.

As shown in figure 1 is the structure of the optical micro cavity of sapphire, it is a Z-type channel with circular section, the radius of which is $200 \mu \mathrm{m}$. The polishing liquid flow into left entrance and flow out from right entrance with a certain speed. After designing the structure of micro cavity, it can work to build 3D structure model of fluid region, divide grid and set up boundary condition[1,2] with a software named Gambit. The flow velocity of polishing liquid that flow into the micro cavity decides the pressure of inner surface of the micro cavity. So the entrance velocity is setted up as boundary condition. And importing the model with Fluent to simulate is the next step.

The motion state of abrasive is influenced by the fluid parameters of polishing liquid, so in order to explore the effect law of polishing abrasive, it needs to explore the influence of velocity and the pressure of polishing liquid in the optical micro cavity firstly $[3,4]$.

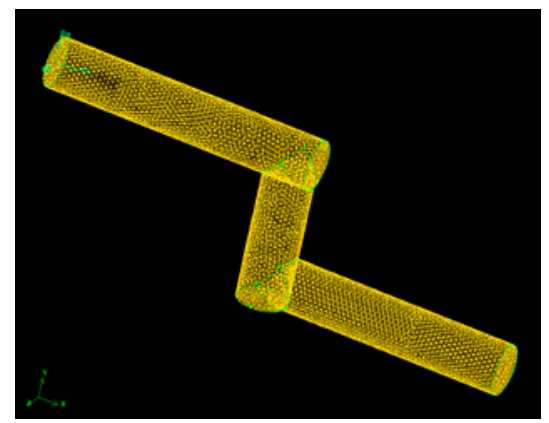

Figure1. The structure after dividing grid

The effect of entrance velocity on the flow velocity in the micro cavity

It needs to set up different entrance velocity in order to explore the effect law of entrance velocity on the flow velocity in the micro cavity., what's more, $v_{1}<v_{2}<v_{5}<v_{4}<v_{9}<v_{\mathbb{L}}$, then figure 2 are the flow velocity distribution in the micro cavity.
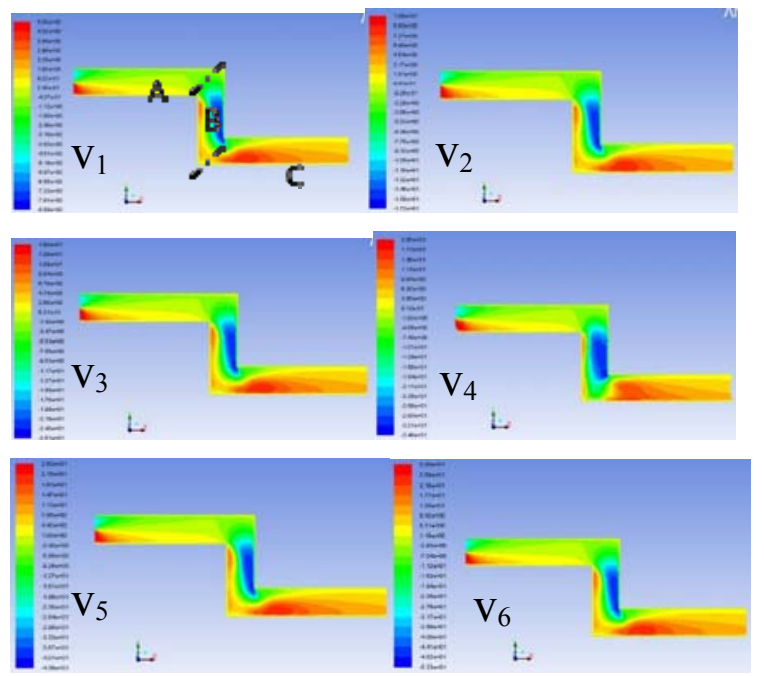

Figure2. The flow velocity of polishing liquid in the micro cavity 
According to the flow velocity distribution in the micro cavity, as the pictures shown, it can be divided into three parts called $\mathrm{A}, \mathrm{B}$ and $\mathrm{C}$. When the entrance velocity are different, the flow velocity distribution in the micro cavity are similar: at part A, the difference of flow velocity at the entrance of the micro cavity is large, which means that the flow velocity above is small and the flow velocity below is large. With the flow of polishing liquid into the micro cavity, the flow velocity begins to be stable, and the difference of flow velocity is small gradually, and it comes to be the most stable state at part A; but when the polishing liquid flow into part B, the flow velocity becomes slow suddenly, and it comes to be the slowest flow velocity in the whole micro cavity, what's more, the difference of left surface and right surface is the largest; At part $\mathrm{C}$, the flow velocity increases suddenly, and the difference of flow velocity is smaller compared to part B. The velocity of abrasive decreases dramatically at part $B$, it is because that when the fluid flows through the end of part A, the flow direction changes $90^{\circ}$ suddenly, most abrasive cannot change their direction immediately and strike against the inner surface with high velocity, which results in the decrease of velocity and the flow along the surface. However the decrease of velocity is not good for polishing.

The sudden increase of velocity can increase the impact of abrasive on the inner surface. The advantage of this kind of impact is that it can increase the efficiency of cutting material here, but because of the large difference of all the velocity of inner surface, the removal amount of every part is different, which results the low polishing effect.

\section{The effect of entrance velocity on the pressure of cavity inner surface}

The fluid in the optical micro cavity, pressure is another essential factor of cutting material besides flow velocity. So it is also important to explore the pressure in the micro cavity. As figure 3 shown below is the results of analysis with Fluent.

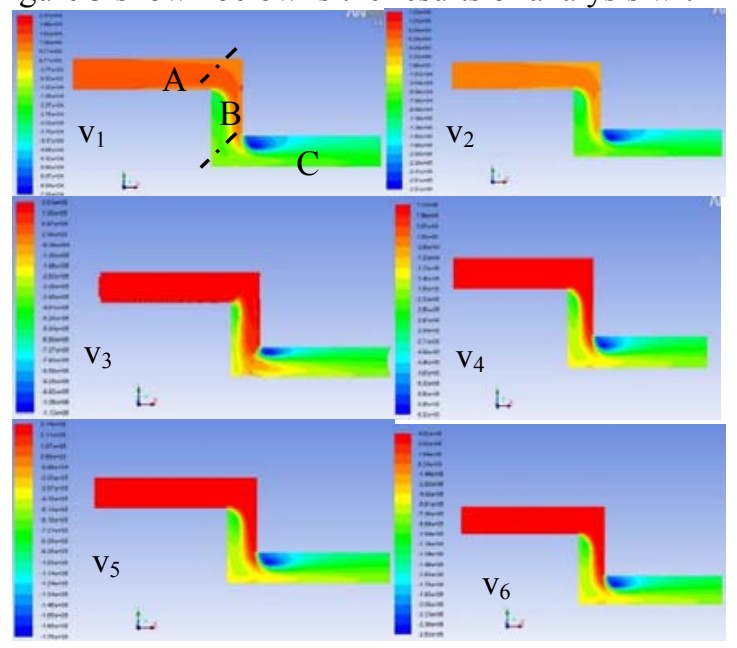

Figure3. The pressure of polishing liquid in the micro cavity

Through analysis, it can be seen that the pressure around the entrance is uniform, but with the entrance into the micro cavity of the fluid, when it enters into part B, the pressure around left inner surface decreases suddenly, which results in the large difference with right inner surface. But part $\mathrm{C}$, which is beneath the highest pressure of right inner surface, the pressure decrease at a high rate of speed and it becomes the lowest pressure in the micro cavity. This is because of the occlusion structure around the corner that make the pressure at this part to decrease. Behind this part, the pressure difference is smaller, but the pressure is lower than entrance pressure obviously.

With the increase of the entrance pressure, the pressure distribution in the micro cavity is also different. When entrance velocity is low $(\mathrm{v} 1 、 \mathrm{v} 2)$, the pressure of part A around the entrance is low, however, while the entrance flow velocity increasing from $\mathrm{v} 3$ to $\mathrm{v} 6$, the pressure of fluid in the micro cavity increase dramatically. At part B, on the corner of Z-type channel, the pressure decrease obviously, which reach the lowest pressure on the second corner. But with the increase of the entrance velocity, this part decrease gradually. At part C, with the increase of entrance velocity, the pressure difference of upper and lower inner surface at the rear end of the micro cavity increase. The increase of pressure difference can result in abrasive and material cutting irregular and inhomogeneous easily.

\section{Discrete phase numerical mimic of abrasive trajectory}

Besides solving the problems of fluid flow, Fluent can also solute equations of continuous phase fluid flow and the solution also contains something about multiphase flow of discrete phase such as particle, water droplets and air bubble [1]. According to the process of solving by Fluent, figure 5 shows the relationship between motion trajectory of abrasive and velocity in different entrance velocity.

It is clear through the motion trajectory of abrasive that the flow velocity of abrasive and polishing liquid are roughly the same. The motion trajectory of abrasive distribute uniformly in the micro cavity near the entrance. But there is almost no motion trajectory of abrasive in the rear of the first right-angle bend. When the velocity is $\mathrm{v} 1$ and $\mathrm{v} 2$ respectively, there is still motion trajectory in the rear of the second corner, but it decrease dramatically when the entrance increase gradually, and there is even a 'valence hole'. The first reason is the shape of the micro cavity, the second one is that with the increase of the entrance velocity of polishing liquid, abrasive is derived by polishing liquid to go through the corridor rapidly, which decrease the impact on the inner surface of the rear cavity. In the rear of the micro cavity, the motion trajectory below is dense and that below is sparse. This kind of condition can influence the removel amount of material in the rear of the cavity, which results the decrease of polishing efficiency and inhomogeneous polishing effect. 

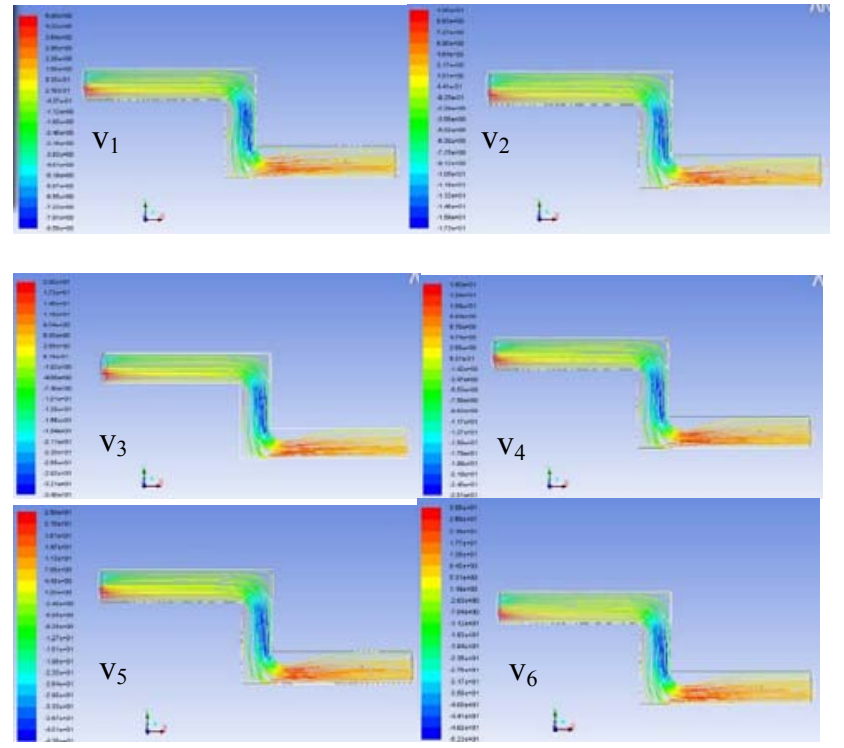

Fig.4 The motion trajectory of abrasive in the micro cavity

\section{The effect of ultrasonic vibration on the polishing effect}

There is no ultrasonic vibration at first, and after polishing liquid flowing through the inside of the micro cavity, impose movement to mimic the effect law of flow velocity and pressure of polishing liquid and the motion trajectory of abrasive when imposing ultrasonic vibration on the micro cavity model. In order to simplify the model, the motion velocity of the micro cavity is transient velocity here.

As figure 5 shown, after imposing vibration in the $x-$ direction, the velocity difference in the micro cavity decrease dramatically and the flow velocity everywhere get to be uniform. But at part B, there is obvious pressure difference of left and right inner surface. And after imposing vibration in the $y$-direction, there is still large velocity difference at part B, but the flow velocity near the inner surface increase obviously, which illustrates that ultrasonic vibration can ameliorate the flow distribution in the micro cavity. But imposing ultrasonic vibration in the $y$-direction is a favor to the impact of polishing abrasive and the inner surface and it is also a favor for polishing.

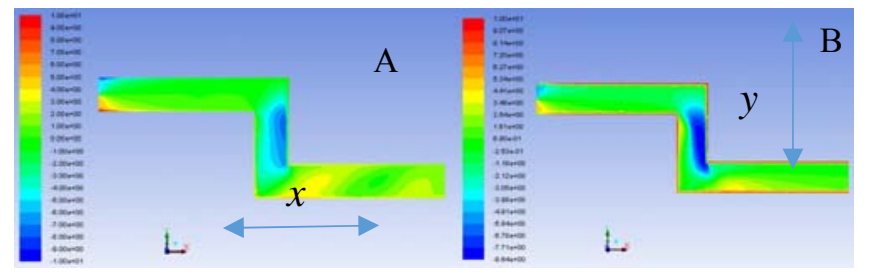

Fig.5 x-direction (A), y-direction (B) the flow velocity in the micro cavity with vibration

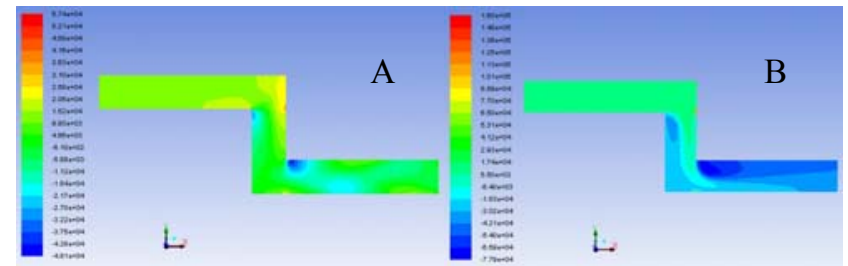

Fig.6 x-direction (A), y-direction (B) the pressure in the micro cavity with vibration

As figure 6 shown, after imposing vibration in the $\mathrm{x}$ and $y$-direction, the pressure difference also decrease, but the pressure in the rear of the second corner is still low. If the vibration is in the $\mathrm{x}$-direction, the pressure of local region is either high or low, and it is not uniform. If the vibration is in the y-direction, the pressure difference of entrance and exit is obvious. This kind of phenomenon illustrates that ultrasonic vibration can ameliorate the pressure in the micro, but the effect is not obvious compared with flow velocity.

It is clear through comparing motion trajectory of abrasive that it can be more uniform when imposing ultrasonic vibration in the $\mathrm{x}$ and $\mathrm{y}$-direction respectively. If the vibration is in the $\mathrm{x}$-direction, there is motion trajectory both in the rear of the first and the second corner in the micro cavity, and the 'valence hole' at the second corner get small dramatically; if the vibration is in the y-direction, the 'valence hole' at the second corner even get vanished. When there is no ultrasonic vibration or the vibration in the $\mathrm{x}$-direction, the motion trajectory is parallel, but when the vibration is in the $y$ direction, some abrasive begin to move from lower end to upper end of the cavity, the motion trajectory get to be more complicated. Just as figure 7 shown.

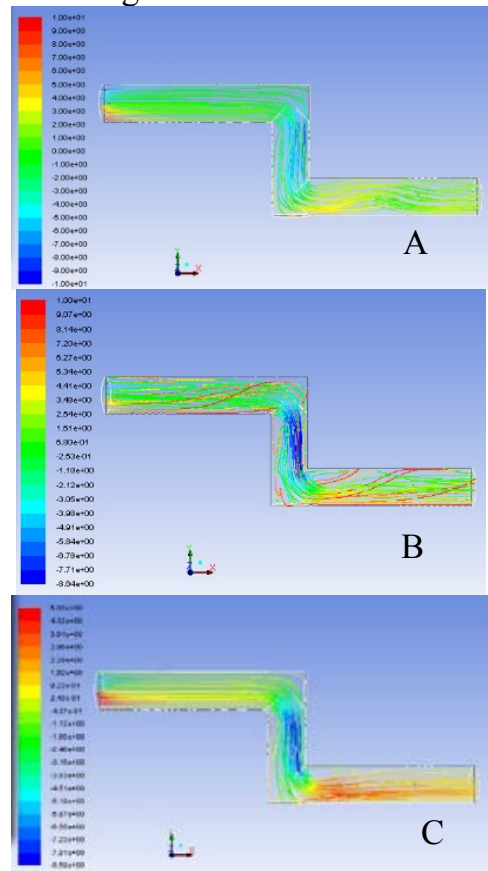

Fig.7 x-direction (A), y-direction (B) and no vibration (C) the motion trajectory of abrasive in the micro cavity 


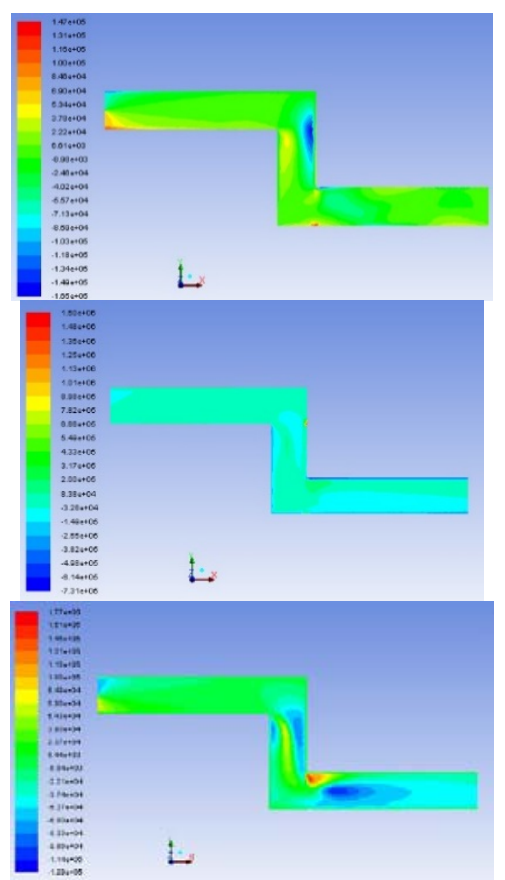

Fig. $8 \mathrm{x}$-direction (A), y-direction (B) and no vibration

(C) material removal amount in the micro cavity

As figure 8 shown, after disposal, it can work to set up material removal amount function to get the figure of removal amount, through which we can see that material removal amount become more uniform after imposing vibration. This phenomenon illustrates that ultrasonic vibration can ameliorate polishing effect and make it more uniform.

\section{CONCLUSIONS}

This artical analyzes the effect law of entrance velocity of polishing liquid on the flow velocity, pressure and the motion trajectory of abrasive in the micro cavity, in this condition, the distribution of flow velocity and pressure is uneven, and there may be 'valence hole' of motion trajectory. But if there is ultrasonic vibration in the $\mathrm{x}$ and $\mathrm{y}$-direction, the pressure and flow velocity become more and more uniform, abrasive can move fully in the micro cavity, the motion trajectory gets to be more complicated, the 'valence hole' at the second corner even get vanished, which ameliorate polishing effect dramatically.

\section{ACKNOWLEDGEMENT}

This work was jointly supported the Natural Science Foundation of China (No. 51305162)

\section{References}

[1] Yong Yu, Introduction and advanced turorial of Flunt[M], Beijing Institute of Technology Press, 2008.

[2] Hongjun Zhu, Fluid analysis and simulation practical turorial of Flunt[M], Posts and Telecommunications Press, 2010.

[3] Junye Li, Micro-hole Abrasive Flow Polishing Device Design and Technology Research[D], Changchun University of Science and Technology , 2011.

[4] Zhengwei Wang, Abrasive Flow Analysis of Acoustic Levitation Polishing and Experimental Raseasch[D],Zhejiang University of Technology, 2012. 